Revista de Derecho

\title{
El principio de precaución en salud como pilar del control constitucional en Colombia
}

The Precautionary Principle in Health as a Pillar of Constitutional Control in Colombia

\section{Camilo Ernesto Ortega Rodríguez}

Abogado independiente

San Juan de Pasto, Colombia

co.24@outlook.com

ORCID: 0000-0003-2774-8214

Artículo de investigación

DOI: https://doi.org/10.32719/26312484.2021.36.7

Fecha de recepción: 19 de octubre de 2020

Fecha de revisión: 19 de enero de 2021

Fecha de aceptación: 16 de marzo de 2021

Fecha de publicación: 1 de julio de 2021 


\section{RESUMEN}

El artículo realiza un comentario a la jurisprudencia del principio de precaución sobre el derecho a la salud. Lo anterior, con el fin de identificar una base constitucional que permita la implementación de distintas medidas de protección a pesar de no contar con una certeza científica del cien por ciento en determinados casos. Para ello, se utilizará un método dogmático no formal, centrando el estudio de la problemática desde un punto de vista teórico, pero manteniendo un contacto con la realidad fáctica y social del problema. Como técnica se usa la recolección de datos, principalmente, la jurisprudencia de la Corte Constitucional de Colombia y artículos académicos que permitan contextualizar el desarrollo del principio. El resultado de la investigación permite observar la aplicación del principio de precaución en salud por el uso del glifosato o por el transporte de carbón, así mismo, ante explotación de hidrocarburos e incluso ante la instalación de antenas de telefonía móvil. En el artículo se concluye que, ante la imprevisibilidad del desarrollo científico, lo pertinente es aplicar las medidas necesarias que impidan la ocurrencia del daño o el riesgo de los derechos, para lo cual el principio de precaución es la base más sólida y racional a desarrollar.

PalABRAS CLAVE: derecho, precaución, protección, salud, ambiente, Constitución, jurisprudencia, casuística.

\section{ABSTRACT}

The article comments on the jurisprudence of the precautionary principle on the right to health. This is done in order to identify a constitutional basis that allows for the implementation of various protective measures, despite the lack of 100 percent scientific certainty in some cases. For this purpose, a dogmatic and nonformal method will be used, focusing the study of the problem from a theoretical point of view, but maintaining contact with the factual and social reality of the problem. As a technique, data collection is used, mainly the jurisprudence of the Colombian Constitutional Court and academic articles that allow for the contextualization of the development of the principle. The result of the research allows us to observe the application of the precautionary principle in health due to the use of glyphosate or the transport of coal, as well as in the face of hydrocarbon exploitation and even the installation of mobile telephone antennas. The article concludes that, in view of the unpredictability of scientific development, the pertinent thing to do is to apply the necessary measures to prevent the occurrence of damage or risk to rights, for which the precautionary principle is the most solid and rational basis to be developed.

KEYWORDs: law, precaution, protection, health, environment, Constitution, jurisprudence, casuistry. 


\section{EL DERECHO A LA SALUD EN LA JURISPRUDENCIA DE LA CORTE CONSTITUCIONAL DE COLOMBIA}

$\mathrm{T}$ emas como la implementación del empaquetado genérico en los productos derivados del tabaco o en los productos azucarados, la prohibición del uso de determinados plaguicidas a raíz de sus efectos tóxicos, la prohibición de productos cosméticos e íntimos que pueden ocasionar cáncer, e incluso, el cierre de aeropuertos para evitar la propagación de enfermedades, son acciones que no pueden fundamentarse en una certeza cien por ciento absoluta desde una perspectiva científica. Lo anterior no se debe a la falta de estudios o evidencia, sino a la existencia de estudios contradictorios o evidencia parcializada que permita tomar una decisión sobre la base de una certeza absoluta de efectividad. En esta perspectiva, se requiere de un instrumento jurídico que permita la actuación del Estado, a través de todas sus instituciones, sin que ello conlleve la implementación de la arbitrariedad y el abuso. El principio de precaución, surgido en materia ambiental, aparece como un nuevo punto de partida en el desarrollo y protección del derecho a la salud, pues ante la falta de certeza científica lo apropiado no es la omisión, sino por el contrario, la acción que permita evitar los peores resultados. No se trata de una función totalmente nueva, sino por el contrario, se trata de una función que se desprende del contenido esencial de la Constitución como mecanismo de control de poder, en un principio frente al poder del Estado y actualmente, frente al poder avasallador de la tecnología. ${ }^{1}$ Por último, la novedad del principio de precaución en materia de salud no se fundamenta en ausencia de anteriores sentencias sobre el tema, sino su eventual independencia frente a una afectación del medio ambiente.

Se procede entonces a estudiar la figura partiendo del derecho a la salud, para luego centrarse en el contenido del principio de precaución y los casos en los que se ha dado aplicación en pro de proteger la vida y la salud de los ciudadanos. En un principio se observó al derecho a la salud como "un derecho fundamental autónomo, en conexidad y directa afectación con un derecho de mayor jerarquía, o por tratarse de un sujeto de especial protección constitucional". ${ }^{2}$ No obstante, la jurisprudencia se fue modificando y se pasó de una visión centrada en la salud física del individuo a una

1. Juan Fernando Jaramillo Pérez et al., El derecho frente al poder: surgimiento, desarrollo y crítica del constitucionalismo moderno (Bogotá: Universidad Nacional de Colombia, 2018), 25.

2. Carlos A. Gómez García y Alejandro Builes Velásquez, "El derecho fundamental a la salud y la política de acceso al sistema: una mirada desde la Ley Estatutaria 1751 del año 2015", Revista de la Facultad de Derecho y Ciencias Politicas-UPB 48, n. ${ }^{\circ} 128$ (2018): 139, https://doi.org/10.18566/rfdcp.v48n128.a06. 
concepción que entienda los aspectos integrales del ser humano. ${ }^{3}$ Asimismo, la jurisprudencia constitucional del derecho a la salud ha ocupado un lugar preponderante en los últimos años. Prueba de ello es que el derecho a la salud ha atravesado 3 facetas en la Corte Constitucional de Colombia

La primera ha sido estableciendo su relación de conexidad con el derecho a la vida, el derecho a la integridad personal y el derecho a la dignidad humana, lo cual le ha permitido a la Corte identificar aspectos del núcleo esencial del derecho a la salud y admitir su tutelabilidad; la segunda ha sido reconociendo su naturaleza fundamental en contextos donde el tutelante es un sujeto de especial protección, lo cual ha llevado a la Corte a asegurar que un cierto ámbito de servicios de salud requeridos sea efectivamente garantizado; la tercera, es afirmando en general la fundamentalidad del derecho a la salud en lo que respecta a un ámbito básico, el cual coincide con los servicios contemplados por la Constitución, el bloque de constitucionalidad, la ley y los planes obligatorios de salud, con las extensiones necesarias para proteger una vida digna. A continuación, pasa la Corte a delimitar y caracterizar el derecho a la salud, en los términos en que ha sido consignado por la Constitución, el bloque de constitucionalidad, la Ley y la jurisprudencia. ${ }^{4}$

Consecuencia de lo anterior es que la protección de la salud de los ciudadanos sea uno de los principales objetivos del Estado social de derecho, ello por cuanto, a través de su implementación se busca el mejoramiento de la calidad de vida de los habitantes, toda vez que

la atención de la salud de las personas residentes en Colombia constituye un cometido programático de carácter social a cargo del Estado; que, sin duda le impone al poder público la misión constitucional concreta de organizar, dirigir y reglamentar, conforme a la ley y a los principios de la función administrativa y atendiendo a los derechos sociales señalados en la Carta Política, un sistema prestacional de seguridad social en materia de salud que comprende, por extensión, la protección de los derechos constitucionales a la vida y a la integridad física. ${ }^{5}$

Asimismo, su objeto conlleva la aplicación de una serie de medidas negativas y positivas. Las primeras denominadas de no interferencia y las segundas aquellas que conllevan la realización de una acción, así

3. Jaime León Gañan, "Hacia un nuevo concepto de la inspección, vigilancia y control del derecho a la salud y del Sistema General de Seguridad Social en Salud en Colombia”, Páginas de Seguridad Social 1, n. ${ }^{\circ} 1$ (2017): 125, https://doi.org/10.18601/25390406.n1.05.

4. Colombia Corte Constitucional, "Sentencia", en Juicio $n .{ }^{\circ}$ T-760/08, 31 de julio de 2008, 24-5.

5. Colombia Corte Constitucional, "Sentencia", en Juicio $n .^{\circ} T-645 / 98,9$ de noviembre de 1998, 6. 
Las libertades y derechos que se mencionan implican obligaciones negativas y positivas. Las obligaciones negativas son de no interferencia de terceros respecto de la libertad sexual o genésica del titular, respecto de su cuerpo, de tratamientos o experimentos no consensuados, etc. Entre las obligaciones positivas se menciona el proporcionar un sistema de protección que permita disfrutar el más alto nivel de salud posible. ${ }^{6}$

Ahora bien, bajo el entendido de considerar al derecho a la salud como un derecho fundamental, se le han asignado al mismo las características de autónomo e irrenunciable, en tanto derecho individual y colectivo. ${ }^{7}$ Ello con el fin de fortalecer su implementación como derecho, al tiempo que fortalecía su carácter fundamental en el ordenamiento jurídico. En ese orden de ideas, el derecho a la salud se constituye como un objetivo a alcanzar en cabeza del Estado, en consecuencia, debe orientar el cumplimiento de dicho fin con todos los medios a su disposición, buscando siempre el mejor resultado posible a la luz del derecho nacional e internacional, este último representado mediante el bloque de constitucionalidad. ${ }^{8}$ De manera adicional, es pertinente recalcar el estatus de protección reforzado que han obtenido los sujetos de especial protección así, "quienes se encuentran en particulares condiciones económicas, físicas o mentales, como los niños, discapacitados y adultos mayores, entre otros, son objeto de especial protección por parte del Estado y de garantía en materia de salud". 9 Ello en tanto su amparo se ve reforzado por sus condiciones particulares o en casos de debilidad manifiesta, ${ }^{10}$ así

los servicios de salud i) deben ser accesibles, de hecho y de derecho, a los sectores más vulnerables y marginados de la población, sin discriminación alguna por cualquiera de los motivos prohibidos; ii) deben estar al alcance geográfico de todos los sectores de la población, en especial los grupos vulnerables o marginados, como las minorías étnicas y poblaciones indígenas, las mujeres, los niños, los adolescentes, las personas mayores, las personas con discapacidades y las personas con VIH/SIDA; iii)los pagos por servicios de atención de la salud y servicios relacionados con los factores determinantes básicos de la salud deberán basarse en el principio de la equidad, a fin de asegurar que esos servicios, sean públicos o privados, estén al alcance de todos, incluidos los grupos socialmente desfavorecidos; y iv) se debe asegurar el derecho a solicitar, recibir y difundir información e ideas acerca de las cuestiones relacionadas con la salud. ${ }^{11}$

6. Rodolfo Figueroa García Huidobro, "El derecho a la salud", Estudios Constitucionales 2 (2013): 302, https://doi.org/ 10.4067/S0718-52002013000200008.

7. Colombia Corte Constitucional, "Sentencia", en Juicio n. ${ }^{\circ} T-313 / 14,29$ de mayo de 2014, 252.

8. Colombia Corte Constitucional, "Sentencia", en Juicio n. ${ }^{\circ} T-454 / 14,1$ de diciembre de 2014, 27.

9. Colombia Corte Constitucional, "Sentencia", en Juicio n. ${ }^{\circ} T-920 / 14,1$ de diciembre de 2014, 27.

10. Colombia Corte Constitucional, "Sentencia", en Juicio n. ${ }^{\circ} T-799 / 13,12$ de noviembre de 2013, 22.

11. Colombia Corte Constitucional, "Sentencia", en Juicio n. ${ }^{\circ}$ T-592/17, 7 de julio de 2014, 17. 
Ahora bien, a pesar de su carácter fundamental y de las medidas reforzadas sobre los sujetos de especial protección, el derecho a la salud ostenta distintas dificultades en la práctica, principalmente la constante aparición de riesgos o peligros que pueden atentar en su contra con ocasión de los nuevos desarrollos y prácticas de producción. En consecuencia, dentro de la jurisprudencia de la Corte, surge la implementación del principio de precaución dentro del derecho a la salud como medida para evitar la ocurrencia de daños ante el constante e imprevisible avance de la sociedad humana.

\section{EL PRINCIPIO DE PRECAUCIÓN EN LA JURISPRUDENCIA COLOMBIANA}

Sea lo primero establecer que el principio de precaución tiene su origen en la protección del medio ambiente. En específico, en países europeos donde la conciencia ambiental comenzó a despertarse luego de los estragos causados después de la segunda guerra mundial. ${ }^{12}$ No obstante su aplicación indirecta desde 1992 en la jurisprudencia colombiana, lo cierto es que solo en años posteriores se realizó su consagración directa como principio que orienta la actuación del Estado. ${ }^{13}$ En ese sentido, el principio funciona en tanto

el riesgo o la magnitud del daño producido o que puede sobrevenir no son conocidos con anticipación, porque no hay manera de establecer, a mediano o largo plazo, los efectos de una acción, lo cual por ejemplo, tiene su causa en los límites del conocimiento científico que no permiten adquirir la certeza acerca de las precisas consecuencias de alguna situación o actividad, aunque se sepa que los efectos son nocivos. ${ }^{14}$

Bajo las anteriores circunstancias, se aplican las medidas de protección necesarias para amparar la protección del ambiente, los derechos de las comunidades y la protección de la salud de las personas. Tal es así que su función es "supera[r] los límites de la ciencia, y protege[r] al medio ambiente y la salud humana más allá de un nexo de causalidad entre dos situaciones". ${ }^{15}$ Es preciso no confundir el principio de precaución con una medida cautelar, la última es un instrumento para "materializar las

12. Carlos Ignacio González-Arruti, "El derecho internacional del medio ambiente y el principio de precaución: una atención a los organismos vivos modificados (OVM)", Dikaion 24, n. ${ }^{\circ} 2$ (2015): 313, https://doi. org/10.5294/dika.2015.24.2.5.

13. Elías Alfonso Buitrago Dangond, "El principio de precaución en la jurisprudencia constitucional”, Derectum 3, n. ${ }^{\circ} 2$ (2018): 111, https://doi.org/10.18041/2538-9505/derectum.2.2018.5213.

14. Colombia Corte Constitucional, "Sentencia", en Juicio $n .{ }^{\circ}$ C-703/10, 6 de septiembre de $2010,40$.

15. Andrea Carolina Ariza Sánchez, "Principio de precaución en la acción de tutela. Casos ambientales Colombianos", Jurídicas CUC 16, n. ${ }^{\circ} 1$ (2020): 197-224, https://doi.org/10.17981/juridcuc.16.1.2020.08. 
determinaciones que al ser adoptadas en la sentencia, reconozcan los pedimentos del demandante" ${ }^{16}$ La diferencia fundamental es la finalidad, si bien en los dos casos se pretende evitar la ocurrencia de un perjuicio, la incertidumbre de la medida cautelar radica en el resultado de un proceso judicial. Por su parte, el principio de precaución actúa ante una incertidumbre pero, contrario a la medida cautelar, su vigencia no depende del resultado de un juicio, puesto que la medida que soluciona se toma desde el fondo de la acción de tutela y tiene carácter permanente.

Por otra parte, si bien cabe mencionar la existencia de medidas previas en cabeza de las administración pública, las mismas no deben confundirse con la aplicación del principio de precaución en material judicial. La diferencia es clara en tanto las medidas previas pueden ser aplicadas ante la existencia de un posible daño o ante la ausencia total del mismo, es decir, la naturaleza de la administración pública es tal que su manejo de los recursos no obedece únicamente a la existencia de riesgos o daños. Por ese motivo, el margen de aplicación es sumamente amplio. Por el contrario, en caso del principio de precaución, su impacto surge al desprender al juez del deber absoluto y tajante de fallar sobre una base probatoria certera, en consecuencia, si bien al matizarse ese deber tiene mayor margen de actuación, nunca llega a tener, ni por asomo, la libertad de la administración pública para ejercer sus facultades.

Desde un punto de vista doctrinario, el principio de precaución parte del principio de responsabilidad del Estado, según el cual las autoridades deben responder por sus omisiones. ${ }^{17}$ En ese sentido, el principio de precaución busca que las omisiones no se consoliden ante un peligro o riesgo que pueda ser evitado, sino que por el contrario, se tomen las medidas previas necesarias para evitar la afectación o el daño. Del mismo modo, el principio de precaución funciona como mecanismo para redirigir los gastos y costos asociados a la ocurrencia de daños o perjuicios en favor del gasto público social prioritario, por tal motivo, su efectividad es doble, en tanto previene los efectos negativos de una situación futura incierta al tiempo que genera ahorro en el funcionamiento del Estado y sus instituciones. ${ }^{18}$ Finalmente, en lo que a principios se refiere, vale la pena mencionar que el principio de precaución es una manifestación absoluta del principio de economía procesal, por cuanto con el mismo se alcanza obtener el mayor resultado con el menor esfuerzo posible, que para el caso en concreto consiste en la prevención del litigio como tal, en tanto es reemplazado por la presentación de una acción de tutela o por la expedición de un acto administrativo. ${ }^{19}$ En ese sentido

16. Jorge Forero Silva, Medidas cautelares en el Código General del Proceso (Bogotá: Editorial Temis, 2020$), 3$.

17. Luis Roberto Ortiz Arciniegas y Lucelly Rocío Munar Castellanos, Derecho Procesal Administrativo y de lo Contencioso Administrativo (Bogotá: Editorial Temis, 2020), 8.

18. Juan Camilo Restrepo, Hacienda Pública (Bogotá: Universidad Externado de Colombia, 2012), 138.

19. Jaime Azula Camacho, Manual de Derecho Procesal (Bogotá: Editorial Temis, 2019), 105. 
los principios del ordenamiento jurídico refuerzan la aplicación del principio y su finalidad.

Por todo lo anterior, el principio de precaución, permite "manejar la incertidumbre propia de las causales y relaciones que pueden estar fuera del control humano y que son básicamente las vinculadas a las ciencias naturales, incluyendo dentro de ellas, las que se refieren a la protección del bien superior de la vida y la salud humana". ${ }^{20}$ Lo anterior se ha aplicado en la jurisprudencia de Corte Constitucional sobre la base de los siguientes requisitos

(i) El principio de precaución se aplica cuando el riesgo o la magnitud del daño generado o que puede sobrevenir no son conocidos con anticipación, porque no hay manera de establecer, a mediano o largo plazo, los efectos de una acción, lo cual generalmente ocurre por la falta de certeza científica absoluta acerca de las precisas consecuencias de un fenómeno, un producto o un proceso; (ii) según los instrumentos internacionales, las normas y jurisprudencia nacionales, el principio de precaución puede ser empleado para proteger el derecho a la salud. ${ }^{21}$

En ese orden de ideas, no es posible la aplicación del principio de precaución ante la ausencia de un indicio científico, pues su objetivo es flexibilizar "el rigor científico que se exige para que el Estado adopte una determinación. [...] se aplica [el principio] en los casos en que es posible conocer las consecuencias sobre el medio ambiente". ${ }^{22}$ No obstante, no es una regla que dé vía libre al ejercicio autónomo de decisiones sin un soporte científico, por cuanto las bases científicas exigidas requieren dar lugar a la posible ocurrencia de una afectación.

De otro lado, se tiene que el principio de precaución no se limita a la posible ocurrencia de un daño, por cuanto "no responde exclusivamente al peligro, noción que se refiere exclusivamente a una posibilidad de daño. Este [principio] responde más bien al riesgo, es decir, a un cierto grado de probabilidad de un daño, en las situaciones en que la magnitud de dicha probabilidad no se ha podido establecer con certeza". ${ }^{23}$ Bajo ese precepto, el riesgo, entendido como concepto más amplio, les permite a los operadores jurídicos valorar cada situación en concreto. Por ese motivo, pueden tomarse distintas determinaciones sin que se haya establecido una tarifa probatoria mínima al respecto, pues será el criterio del operador el determinante en cada caso.

20. Carmen Artigas, El principio precautorio en el derecho y la politica internacional (Santiago de Chile: Naciones Unidas, 2001), 7.

21. Colombia Corte Constitucional, "Sentencia", en Juicio n. ${ }^{\circ} T-397 / 14,6$ de septiembre de 2010, 68.

22. Colombia Corte Constitucional, "Sentencia", en Juicio n. ${ }^{\circ} C-449 / 15,16$ de julio de 2015, 36.

23. Colombia Corte Constitucional, "Sentencia", en Juicio $n .^{\circ} T-298 / 17,8$ de mayo de 2017, 45. 
Del mismo modo, se tiene que el principio funciona como un instrumento o herramienta que faculta a las autoridades estatales "para actuar ante la afectación, daño, riesgo o peligro que enfrenta el medio ambiente como consecuencia de la ejecución de actividades o proyectos permitidas (sic) que puedan comprometerlo gravemente, al igual que a los derechos con él relacionados". ${ }^{24}$ Motivo por el cual se observa un principio que sirve como pilar en la toma de distintos tipos de decisiones, tanto "la administrativa, como justificación de decisiones nacionales restrictivas con limitado sustento probatorio; y la propiamente jurisdiccional, como herramienta procesal en los contenciosos internacionales donde la aportación probatoria es escasa o contradictoria". ${ }^{25}$ Como consecuencia de lo anterior, la Corte Constitucional reconoce que la ciencia se encuentra siempre en constante desarrollo y, ante la falta de certeza, se impone el deber de actuar con mesura para evitar una posible lesión o daño. ${ }^{26}$ Por ese motivo ha extendido su aplicación teniendo en cuenta el derecho a la salud amparado por la jurisprudencia de la Corte.

Se tiene así, a manera de síntesis, que el principio de precaución actúa ante circunstancias de riesgo para el ambiente o la salud humana, pero solamente si no hay una certeza absoluta sobre los posibles resultados nocivos. En ese sentido, las autoridades tienen el deber de tomar las medidas necesarias para evitar la ocurrencia de afectaciones antes de que sucedan. ${ }^{27}$

\section{CASUÍSTICA DEL DERECHO A LA SALUD Y EL PRINCIPIO DE PRECAUCIÓN}

Partiendo del contenido del derecho a la salud y teniendo en cuenta los avances jurisprudenciales realizados en el principio de precaución, se procede a examinar los casos más representativos en los que el principio ha pasado de un enfoque eminentemente ambiental hasta convertirse en un principio de protección del derecho a la salud. En ese orden de ideas, la mayoría de las sentencias y casos provienen de acciones de tutela, pues la misma se encuentra habilitada para la protección de los derechos bien sea por su violación o ante una eventual amenaza. En ese sentido, la jurispruden-

24. Colombia Corte Constitucional, "Sentencia", en Juicio n. ${ }^{\circ} T-236 / 17,21$ de abril de 2017, 45.

25. Justo Corti Varela, "El Principio de Precaución en la Jurisprudencia Internacional", Revista Española de Derecho Internacional, Sección Estudios 69, n. ${ }^{\circ} 1$ (2017): 221, https://doi.org/10.17103/redi.69.1.2017.1.08.

26. Colombia Corte Constitucional, "Sentencia", en Juicio n. ${ }^{\circ} T-021 / 19,28$ de enero de 2019, 36.

27. Colombia Corte Constitucional, "Sentencia", en Juicio n. ${ }^{\circ} T-614 / 19,16$ de diciembre de 2019, 81. 
cia cumple su papel como un criterio vinculante por analogía fáctica que respalda la implementación constante del derecho en la sociedad contemporánea. ${ }^{28}$

Se observa una gran variedad de casos con supuestos fácticos diferentes que permiten constituir una base jurisprudencial sólida para aplicar el principio de precaución de una nueva manera. ${ }^{29}$ Además, se observa el uso de la ponderación, entendida como "una exigencia de proporcionalidad que implica un orden de preferencia relativo al caso concreto". ${ }^{30}$ En ese sentido, se observa que su uso ha ayudado al establecimiento de una serie de sentencias que otorgan vigencia al principio de precaución.

La contaminación a causa de los ferrocarriles. En el caso en concreto, se adelantó acción de tutela con ocasión del ruido causado por un tren cercano a la vivienda de los accionantes. En el caso, se alegó que, al transportar carbón, el tren representaba un riesgo para la salud de los habitantes y que, además, generaba una fuente insoportable de ruido que afectaba su calidad de vida. En el caso, la Corte concedió la razón a la parte tutelante por cuanto

La actividad de transporte férreo, al poder generar menoscabo en el medio ambiente, está sujeta al principio de precaución. Este se aplica cuando - aunque haya un principio de certeza técnica- existe incertidumbre científica respecto de los efectos nocivos de una medida o actividad. En ese caso, debe preferirse la solución que evite el daño y no aquella que pueda permitirla. El principio no solo está concebido para proteger el derecho al medio ambiente sino también el derecho a la salud. Así, cuando por causa del trasporte ferroviario exista contaminación por ruido o por emisión de partículas de carbono, en caso de que exista duda razonable respecto de si estas afectan el entorno natural o la salud de las personas, deben tomarse las medidas que anticipen y eviten cualquier daño. ${ }^{31}$

En ese orden de ideas, se ordenó el cese del transporte entre las 10 y 30 de la noche y las 4 y 30 de la mañana, además, se ordenó controlar el nivel de contaminación a causa del trasporte de carbón en el lugar. Se tiene entonces una aplicación del principio de precaución sobre la base de la contaminación auditiva y sobre el posible riesgo que acarrea la movilización del carbón en la salud de los habitantes. Es por tanto, un antecedente de que las actividades empresariales deben ceder ante el bienestar de la comunidad y su protección.

28. Diego Eduardo López Medina, El derecho de los jueces (Bogotá: Legis Editores, 2006), 134.

29. Manuel Fernando Quinche Ramírez, Vías de hecho. Acción de tutela contra providencias (Bogotá: Editorial Temis, 2020), 17.

30. Luis Prieto Sanchíz, "El juicio de ponderación constitucional", en El principio de proporcionalidad en el Estado constitucional, ed. Miguel Carbonell (Bogotá: Universidad Externado de Colombia, 2007), 120.

31. Colombia Corte Constitucional, "Sentencia", en Juicio n. ${ }^{\circ}$ T-672/14, 10 de septiembre de 2014, 25. 
El riesgo asociado con la aspersión de glifosato. En el presente caso, se analizó la acción de tutela adelantada en contra de las fumigaciones con glifosato, mismas realizadas con ocasión de la lucha que vive el país en contra del narcotráfico. El sujeto de especial protección para el caso en concreto fue una comunidad indígena, al respecto, la Corte consideró que

[a]1 ser la actividad de aspersión aérea de cultivos ilícitos con glifosato, una actividad que como se ha visto hasta ahora, puede llegar a generar efectos nocivos no solo en el medio ambiente como entidad autónoma sino además en la salud de las poblaciones humanas, es aplicable a tales impactos el principio de precaución. ${ }^{32}$

Por ende, se ordenó su suspensión, pues no existía certeza sobre los efectos nocivos para la salud de las comunidades indígenas que habitaban la zona. La sentencia significó un cese en la práctica de las aspersiones, pero a su vez, la garantía de que la salud de los habitantes se encuentra por encima de los demás objetivos del Estado, incluso, sobre el de la seguridad y control del territorio. La sentencia significó un cambio, por cuanto con anterioridad la Corte se había limitado a impartir la orden de realizar la consulta previa, sin que ello significara la suspensión inmediata de las fumigaciones. Un extracto de un salvamento de voto muestra el descontento de los magistrados de la época, quienes sostienen que

[s]orprende entonces que ante la claridad de las disposiciones constitucionales mencionadas, y haciendo caso omiso de la legislación vigente y de convenios internacionales suscritos por Colombia, en esta acción de tutela no se haya impartido la orden de suspensión inmediata de la fumigación aérea de los denominados cultivos ilícitos en la Amazonía Colombiana, pese a la existencia de abundantes pruebas que en la propia sentencia se mencionan y analizan según las cuales no se encuentra demostrado que el glifosato no causa daño a los seres humanos, ni a la vida animal, ni a la vida vegetal, ni a los recursos hídricos, sino que por el contrario lo que aparece es que existen daños a la salud de la niñez y de la población adulta con manifestaciones tales como enfermedades de la piel, afectación de las vías respiratorias, alteración de la función digestiva, pérdidas de cosechas de cultivos de pan coger, muerte de animales domésticos, alteración de las aguas para el consumo humano y de los animales. ${ }^{33}$

El principio de precaución se muestra así como un nuevo pilar de la protección constitucional del derecho a la salud, sin embargo, hasta este punto su alcance se deriva de su aplicación al medio ambiente, del mismo modo que el siguiente caso.

32. Colombia Corte Constitucional, "Sentencia", en Juicio $n .^{\circ} T-080 / 17,7$ de febrero de 2017, 37.

33. Colombia Corte Constitucional, "Sentencia", en Juicio n. ${ }^{\circ} S U-383 / 17,13$ de mayo de 2003, 138. 
La interrupción de actividades mineras extractivas. En un sentido similar, se observa que las operaciones mineras pueden ser detenidas con ocasión del principio de precaución en materia de salud. En el reconocido caso del Río Atrato, el principio de precaución sirvió para dotar a las comunidades de la capacidad de ejercer acciones en pro de su bienestar y del bienestar del ambiente, en ese sentido,

las declaraciones, los informes y los conceptos citados confirman que las actividades contaminantes producidas por la explotación minera ilegal pueden llegar a tener impactos directos sobre la salud de las personas y adicionalmente, otra clase de impactos indirectos sobre el bienestar humano, como la disminución de productos del bosque que afecta el balance alimentario y medicinal, y puede producir cambios en las prácticas tradicionales, usos y costumbres de las comunidades étnicas asociados a la biodiversidad. ${ }^{34}$

En consecuencia, la sentencia considera que la afectación, si bien no es absoluta y consumada, si cuenta con un alto nivel de probabilidad. Por ello que se aplique la protección a la salud en relación con la afectación con el ambiente. No obstante, otros casos muestran una vertiente más independiente de la aplicación de principio, tal y como se ve a continuación.

Los riesgos de las antenas de telefonía móvil. Las sentencias que ordenan la reubicación de antenas de telefonía móvil se remontan a 2001. La acción afirmaba que, a raíz de la instalación de una antena de base de telefonía celular, el estado de salud de la tutelante se había deteriorado. El caso resultó paradigmático por cuanto la Corte encontró probada la causalidad entre las emisiones electromagnéticas y el estado de salud de la accionante. En consecuencia ordenó suspender, de manera transitoria, la operación y trasladar la ubicación de la antena. ${ }^{35}$

Posteriormente, en 2008 se adelantó una acción de tutela que buscaba proteger los derechos de una pareja y sus hijos menores de edad. En esa ocasión se consideró que los niños eran sujetos de especial protección, por tanto, se ordenó la aplicación del principio de precaución para casos similares. Ello por cuanto, sobre el asunto en cuestión se había ocurrido el fenómeno del hecho superado. ${ }^{36}$

No obstante, la jurisprudencia ha establecido límites a la aplicación del principio de precaución en materia de salud. Tal fue el caso de la acción de tutela presentada por una accionante que fue intervenida quirúrgicamente para implantarle un cardio desfibrilador. En el caso, si bien la Corte Constitucional consideró que el principio de precaución no requiere de un grado de certeza absoluta, lo cierto es que si requiere

34. Colombia Corte Constitucional, "Sentencia", en Juicio n. ${ }^{\circ} T-622 / 16,10$ de noviembre de $2016,27$.

35. Colombia Corte Constitucional, "Sentencia", en Juicio n. ${ }^{\circ} T-1062 / 01,11$ de octubre de 2001, 26.

36. Colombia Corte Constitucional, "Sentencia”, en Juicio n. ${ }^{\circ} T-299 / 08,3$ de abril de 2008, 32. 
un indicio probatorio racional indique la posible afectación del derecho a la salud. En consecuencia, los documentos aportados por la accionante no lograron acreditar que la existencia de una antena de radiodifusión cercana a su domicilio pudiese ocasionar un perjuicio o daño al funcionamiento de su implante. Por ese motivo, se negó la protección constitucional pero exhortó al Ministerio de Telecomunicaciones para que analice la situación a la luz del principio de precaución. ${ }^{37}$

La Corte ha ratificado su posición sobre la posibilidad de interferencia de las antenas electromagnéticas sobre mecanismos de funcionamiento cardíacos. En este orden de ideas, en el 2014, confirmó la negativa a conceder el amparo a una persona que se encontraba en similares circunstancias, por cuánto

No se vulnera el derecho fundamental a la salud por la instalación de una antena de telecomunicaciones cuando no hay demostración alguna de la existencia de un peligro, amenaza o afectación del estado de salud del accionante como consecuencia de las radiaciones electromagnéticas que ella emita. La aplicación del principio de precaución requiere que exista peligro del daño, que este sea grave e irreversible y que exista un principio de certeza científica, así no sea absoluta. ${ }^{38}$

En ese sentido, se evidencia que si bien no se requiere una carga probatoria preestablecida, si se requiere la existencia de estudios científicos que acrediten el riesgo afectación que puede causar de no aplicarse el principio de precaución. Del mismo modo, frente al caso de enfermedades coronarias, la Corte sostuvo que "En el caso particular, si bien no hay duda de que los accionantes padecen de enfermedades coronarias que en la actualidad están siendo tratadas, no existen pruebas que demuestren que las mismas pueden verse afectadas" ${ }^{39}$

Por otra parte, en el 2012, se analizó el caso de una menor de edad que instauró acción de tutela con ocasión de la instalación de una antena de telefonía móvil cercana a su residencia. En el caso se alegó que, al sufrir de cáncer, su situación se vería gravemente deteriorada con ocasión de las ondas electromagnéticas emitidas por el mecanismo de comunicación. En dicha sentencia la Corte consideró que

a pesar de que no es posible constatar una relación directa entre las afecciones de salud de las personas y la radiación no ionizante, la clasificación de los campos electromagnéticos de radiofrecuencia como posiblemente carcinógenos para los humanos, permite que las autoridades, en aplicación del principio de precaución, tomen medidas frente a la radiación, con el fin de evitar que se produzcan daños en la salud derivados de los riesgos medioam-

37. Colombia Corte Constitucional, "Sentencia", en Juicio n. ${ }^{\circ} T-360 / 10,11$ de mayo de 2010, 26.

38. Colombia Corte Constitucional, "Sentencia", en Juicio n. ${ }^{\circ} T-701 / 14,15$ de septiembre de $2014,18$.

39. Colombia Corte Constitucional, "Sentencia", en Juicio $n .^{\circ} T-713 / 16,15$ de diciembre de 2016, 60. 
bientales a los que se ven sometidos los accionantes, como consecuencia de la omisión legislativa frente a este tema. ${ }^{40}$

Sobre ese argumento, se ordenó el retiro de la antena y se dio la orden al Ministerio de Tecnologías de la Información y comunicación para que se diseñe un plan de reubicación de las antenas de comunicación, teniendo en cuenta la ubicación de hospitales, viviendas, instituciones educativas u hogares geriátricos. Sin embargo, la jurisprudencia no ha sido constante respecto a retirar antenas cercanas a hogares geriátricos, por cuanto se ha negado el amparo alegando que se trata de un derecho colectivo que no puede ser amparado por vía de tutela. Al respecto, el salvamento de voto expresa que "[e]xiste una omisión en la regulación, toda vez que no se han fijado los límites de ubicación, en términos de distancia, de las antenas de telefonía móvil celular, que eviten los posibles efectos perjudiciales que pueda generar a la salud humana la exposición a esta clase de radiación electromagnética". ${ }^{41}$

Finalmente, el principio de precaución también se implementado al momento de declarar la constitucionalidad de normas que exigen un grado de estudios superior, para aquellas personas que se dedican a su instalación y ubicación en el terreno. Así, la Corte Constitucional consideró que los técnicos no estaban en capacidad adelantar este tipo de funciones por cuánto

[n]o se trata de riesgos eventuales que afecten un área especial de la vida de algunas personas. Se trata por el contrario de riesgos graves a los cuales se ven expuestas prácticamente todas las personas en las diversas actividades de su cotidianeidad. Al no exigir unos requisitos mínimos de formación académica para el desarrollo de la proyección y diseño de instalaciones eléctricas de nivel medio, el Congreso expuso bienes jurídicamente protegidos y de gran valor constitucional a un riesgo social. ${ }^{42}$

Sobre la base de lo anterior, se tiene que la instalación y ubicación de antenas electromagnéticas puede vulnerar el derecho a la salud de los habitantes tomando como base el principio de precaución. En ese orden de ideas, se observa una tendencia mayoritaria al amparo de este derecho ante la existencia de otros fallos que niegan su procedencia, bien sea por falta de material o evidencia probatoria o bien a razón de considerar al derecho a la salud como un derecho colectivo. Esta última interpretación acogida por quienes desconocen el carácter fundamental que se le ha otorgado.

40. Colombia Corte Constitucional, "Sentencia", en Juicio n. ${ }^{\circ} T-1077 / 12,12$ de diciembre de $2012,53$.

41. Colombia Corte Constitucional, "Sentencia", en Juicio n. ${ }^{\circ} T-149 / 15,8$ de abril de 2015, 45.

42. Colombia Corte Constitucional, "Sentencia", en Juicio $n .{ }^{\circ}$ C-166/15, 15 de abril de 2015, 48. 


\section{UNA MIRADA INTEGRAL DEL PRINCIPIO DE PRECAUCIÓN EN SALUD}

Puede observarse, sobre la base de la anterior casuística, que el principio de precaución aplicado al derecho a la salud consiste en un pilar independiente de interpretación constitucional. Es palpable como un principio aplicado, en principio, al ámbito del derecho ambiental, y comienza a desplazarse hacia otros derechos constitucionales. En ese orden de ideas es preciso recalcar que, a diferencia de los casos de glifosato o hidrocarburos, en los asuntos relacionados con la implementación de antenas electromagnéticas puede observarse una ausencia de un componente ambiental predominante. Es decir, tanto en el caso del glifosato como en el de hidrocarburos se observa que existía una fuerte afectación hacia el ambiente y que, como consecuencia de ello, se derivaban una serie de afectaciones a la salud de las personas. Sin embargo, respecto a la implantación de antenas de telefonía móvil se observa que no existe un amplio grado de afectación medioambiental en el contenido de las sentencias, el principal factor de riesgo pasa a ser la salud de las personas, sin que sea necesario para su protección la existencia de un fuerte riesgo al medio ambiente. De este modo, se han evidenciado una serie de antecedentes precisos que justifican la toma de medidas en pro de la protección de la salud de las personas. Dichas medidas, podrán consistir en el cese de las actividades que puedan ocasionar un daño o en el ejercicio de facultades positivas que pueden ayudar a mejorar las condiciones para prevenirlo.

\section{CONCLUSIÓN}

El principio de precaución tiene plena aplicabilidad frente a los riesgos o amenazas que puedan vulnerar el derecho a la salud en Colombia. En ese sentido, se observa que el principio comienza a constituirse como un punto de aplicación autónomo en la jurisprudencia constitucional del derecho a la salud. Se deja de lado la existencia de una afectación medioambiental predominante, para pasar entonces, a considerar a la salud en sí misma como una justificación suficiente para implementar medidas precautorias. Motivo por el cual, el principio de precaución no puede ser considerado ya un tema de aplicabilidad de derecho público ambiental, sino que pasa constituirse como un fundamento de control constitucional independiente sobre la actuación de la administración y de los entes jurisdiccionales.

Debe aclararse que el funcionamiento del principio de precaución se aplica ante la incertidumbre de un daño a la salud. Es decir, tal como se observó en los casos relacionados con el glifosato y las antenas de transmisión, no puede exigirse la certeza absoluta del daño para aplicación de las medidas protectoras, por cuanto la evidencia hasta la fecha no dota a los jueces de dicha información. Del mismo modo, es preciso 
aclarar que el juez no se deja llevar tan solo por una simple opinión para la toma de sus decisiones, sino que por el contrario, toma bases científicas que le permitan, en cada caso, modular su decisión para el asunto en concreto.

El presente artículo se termina en un mundo que trata de afrontar la primera pandemia mundial en el siglo veintiuno, queda por ver si en un futuro los jueces tendrán la potestad de tomar las medidas precautorias necesarias al considerar un posible riesgo en el otro extremo del planeta, y así, tomar las medidas que consideren necesarias. $\mathrm{La}$ precaución pasa a convertirse de una excepción, a un valor fundamental para evitar consecuencias nefastas en la vida, la salud, la economía y la sociedad humana. No obstante, no puede otorgársele un carácter absoluto, por cuanto, se requiere de cierto nivel de evidencia científica para su implementación. Pese a ello, ante un mundo con riesgos imprevisibles, la precaución razonada, justificada y lógica puede ser una valiosa herramienta de protección.

\section{BIBLIOGRAFÍA}

Ariza Sánchez, Andrea Carolina. "Principio de precaución en la acción de tutela. Casos ambientales Colombianos", Jurídicas CUC 16, n. ${ }^{\circ} 1$ (2020): 197-224. https://doi.org/10.17981/ juridcuc.16.1.2020.08.

Artigas, Carmen. El principio precautorio en el derecho y la política internacional. Santiago de Chile: Naciones Unidas, 2001.

Azula Camacho, Jaime. Manual de Derecho Procesal. Bogotá: Editorial Temis, 2019.

Buitrago Dangond, Elías Alfonso. "El Principio de Precaución en la Jurisprudencia Constitucional". Derectum 3, n. ${ }^{\circ} 2$ (2018): 107-26. https://doi.org/10.18041/2538-9505/derectum.2.2018.5213.

Corti Varela, Justo. "El Principio de Precaución en la Jurisprudencia Internacional". Revista Española de Derecho Internacional Sección Estudios 69, n. ${ }^{\circ}$ (2017): 221-43. https://doi. org/10.17103/redi.69.1.2017.1.08.

Forero Silva, Jorge. Medidas cautelares en el Código General del Proceso. Bogotá: Editorial Temis, 2020.

García Huidobro, Rodolfo Figueroa. "El derecho a la salud". Estudios Constitucionales 2 (2013): 283-332. https://doi.org/10.4067/S0718-52002013000200008.

Gómez García, Carlos A., y Alejandro Builes Velásquez. "El derecho fundamental a la salud y la política de acceso al sistema: una mirada desde la Ley Estatutaria 1751 del año 2015". Revista de la Facultad de Derecho y Ciencias Políticas-UPB 48, n. 128 (2018): 135-67. https://doi.org/10.18566/rfdcp.v48n128.a06.

González Arruti, Carlos Ignacio. "El derecho internacional del medio ambiente y el principio de precaución: una atención a los organismos vivos modificados (OVM)". Dikaion 24, n. ${ }^{\circ}$ 2 (2015): 307-35. https://doi.org/10.5294/dika.2015.24.2.5. 
Jaramillo Pérez, Juan Fernando, Mauricio García Villegas, Andrés Abel Rodríguez Villabona y Rodrigo Uprimmy Yepes. El derecho frente al poder: surgimiento, desarrollo y crítica del constitucionalismo moderno. Bogotá: Universidad Nacional de Colombia, 2018.

León Gañan, Jaime. "Hacia un nuevo concepto de la inspección, vigilancia y control del derecho a la salud y del Sistema General de Seguridad Social en Salud en Colombia". Páginas de Seguridad Social 1, n. ${ }^{\circ} 1$ (2017): 123-40. https://doi.org/10.18601/25390406.n1.05.

López Medina, Diego Eduardo. El derecho de los jueces. Bogotá: Legis Editores, 2006.

Munar Castellanos, Lucelly Rocío, y Luis Roberto Ortiz Arciniegas. Derecho Procesal Administrativo y de lo Contencioso Administrativo. Bogotá: Editorial Temis, 2020.

Prieto Sanchíz, Luis. "El juicio de ponderación constitucional”. En El principio de proporcionalidad en el Estado constitucional, editado por Miguel Carbonell, 99-146. Bogotá: Universidad Externado de Colombia, 2007.

Quinche Ramírez, Manuel Fernando. Vias de hecho. Acción de tutela contra providencias. Bogotá: Editorial Temis, 2020.

Restrepo, Juan Camilo. Hacienda Pública. Bogotá: Universidad Externado de Colombia, 2012.

\section{Sentencias}

Colombia Corte Constitucional. "Sentencia”. En Juicio n. ${ }^{\circ}$ T-645/98. 9 de noviembre de 1998.

—. "Sentencia". En Juicio n. ${ }^{\circ}$ T-1062/01. 11 de octubre de 2001.

—. "Sentencia". En Juicio n. ${ }^{\circ} S U-383 / 17.13$ de mayo de 2003.

—. "Sentencia". En Juicio n. ${ }^{\circ}$ T-760/08. 31 de julio de 2008.

—. "Sentencia". En Juicio n. ${ }^{\circ}$ T-299/08. 3 de abril de 2008.

—. "Sentencia". En Juicio n. ${ }^{\circ}$ T-360/10. 11 de mayo de 2010.

—. "Sentencia". En Juicio n. ${ }^{\circ}$ T-397/14. 6 de septiembre de 2010.

—. "Sentencia". En Juicio $n .{ }^{\circ}$ C-703/10. 6 de septiembre de 2010.

—. "Sentencia". En Juicio n. ${ }^{\circ}$ T-1077/12. 12 de diciembre de 2012.

—. "Sentencia". En Juicio n. ${ }^{\circ}$ T-799/13. 12 de noviembre de 2013.

—. "Sentencia". En Juicio n. ${ }^{\circ}$ T-313/14. 29 de mayo de 2014.

—. "Sentencia". En Juicio n. ${ }^{\circ}$ T-454/14. 1 de diciembre de 2014.

—. "Sentencia". En Juicio n. ${ }^{\circ}$ T-592/17. 7 de julio de 2014.

—. "Sentencia". En Juicio n. ${ }^{\circ}$ T-672/14. 10 de septiembre de 2014.

—. "Sentencia". En Juicio n. ${ }^{\circ}$ T-701/14. 15 de septiembre de 2014.

—. "Sentencia". En Juicio n. ${ }^{\circ}$ T-920/14. 1 de diciembre de 2014.

—. "Sentencia". En Juicio n. ${ }^{\circ}$ T-149/15. 8 de abril de 2015.

—. "Sentencia". En Juicio n. ${ }^{o}$ : C-166/15. 15 de abril de 2015. 
FORO 36, julio-diciembre 2021

—. "Sentencia". En Juicio n. ${ }^{\circ}$ C-449/15. 16 de julio de 2015.

—. "Sentencia". En Juicio n. ${ }^{\circ}$ T-622/16. 10 de noviembre de 2016.

—. "Sentencia". En Juicio n. ${ }^{\circ} T-713 / 16.15$ de diciembre de 2016

—. "Sentencia". En Juicio n. ${ }^{\circ} T-080 / 17.7$ de febrero de 2017.

—. "Sentencia". En Juicio n. ${ }^{\circ}$ T-236/17. 21 de abril de 2017.

—. "Sentencia". En Juicio n. ${ }^{\circ}$ T-298/17. 8 de mayo de 2017.

—. "Sentencia". En Juicio n. ${ }^{\circ}$ T-021/19. 28 de enero de 2019.

—. "Sentencia". En Juicio n. ${ }^{\circ}$ T-614/19. 16 de diciembre de 2019. 